VoL. 63 (2001) [195-218]

\title{
ANALYSIS OF THE AFFINE TRANSFORMATIONS OF THE TIME-FREQUENCY PLANE
}

\author{
Filippo De Mari and Krzysztof NowaK
}

\begin{abstract}
We consider two aspects of the action of the extended metaplectic representation of the group $G$ of affine, measure and orientation preserving maps of the timefrequency plane on $L^{2}$ functions on the line. On the one hand, we list, up to equivalence, all possible reproducing formulas that arise by restricting the representation to connected Lie subgroups of $G$. On the other hand, we describe, in terms of Weyl calculus, the commutative von Neumann algebras generated by restriction to one-parameter subgroups.
\end{abstract}

\section{INTRODUCTION AND PRELIMINARIES}

The time-frequency plane consists of pairs of points $(x, \xi), x, \xi \in \mathbb{R}$, where $x$ denotes time and $\xi$ frequency. Its main purpose is to provide time-frequency representations of signals (one wants to identify essential frequencies at every moment of time). The basic objects related to it are the extended metaplectic representation, the Wigner distribution and the Weyl calculus of pseudodifferential operators. In this paper we examine reproducing formulas coming from restrictions of the extended metataplectic representation and commutative von Neumann algebras generated by the values of the extended metaplectic representation on one-parameter subgroups.

We begin by recalling basic operations on functions: time shifts, frequency shifts, Fourier transform, dilations and multiplications by purely imaginary gaussians, and by interpreting them as transformations of the time-frequency plane. Later we define the extended metapletic representation, Wigner distributions, Weyl pseudodifferential operators, and we present their basic properties. At the end of the section we discuss our results.

Let $f \in L^{2}(\mathbb{R})$. The operations $f \mapsto f(\cdot-q), f \mapsto e^{2 \pi i \cdot p} f$ are the time shift by $q$ and the frequency shift by $p$. It is intuitively clear that on the level of time-frequency representations they correspond to the transformations $(x, \xi) \mapsto(x+q, \xi)$, and $(x, \xi) \mapsto$ $(x, \xi+p)$. Denote by $\mathcal{F}$ the Fourier transform $\mathcal{F} f(\xi)=\int_{-\infty}^{\infty} f(x) e^{-2 \pi i x \xi} d x$ and by $g$

Received 9th May, 2000

Partially supported by the TMR Network "Harmonic Analysis", contract N. FMRX-CT97-0159.

We would like to thank G. Mauceri and F. Ricci for useful conversations.

Copyright Clearance Centre, Inc. Serial-fee code: 0004-9727/01 \$A2.00+0.00. 
the Gaussian, normalised in such a way that $\mathcal{F} g=g$ and $\|g\|_{L^{2}(\mathbb{R})}=1$. The function $g$ is concentrated in a small neighbourhood of 0 and so is its Fourier transform. For this reason $g$ may serve as a basic time-frequency block concentrated at the point $(0,0)$, and the function $g_{q, p}(x)=e^{2 \pi i p x} g(x-q)$ may serve the same purpose for the point $(q, p)$. An easy computation shows that $\mathcal{F}^{-1} g_{q, p}=e^{2 \pi i q p} g_{-p, q}$. This means that the inverse Fourier transform corresponds to the transformation $(q, p) \mapsto(-p, q)$, that is, to rotation by $\pi / 2$. Similar arguments show that the time-frequency plane transformations corresponding to $f \mapsto t^{-1 / 2} f(\cdot / t)$, and $f \mapsto e^{\pi i r \cdot{ }^{2}} f(\cdot)$ are $(q, p) \mapsto\left(t q, t^{-1} p\right)$, and $(q, p) \mapsto(q, p+r q)$.

The above transformations of the time-frequency plane generate the group of measure and orientation preserving affine maps. This group may be described as the semidirect product $\mathbb{R}^{2} \rtimes S L(2, \mathbb{R})$, where the group operation is given by the formula

$$
\left(\left[\begin{array}{l}
q_{1} \\
p_{1}
\end{array}\right], A_{1}\right)\left(\left[\begin{array}{l}
q_{2} \\
p_{2}
\end{array}\right], A_{2}\right)=\left(\left[\begin{array}{l}
q_{1} \\
p_{1}
\end{array}\right]+A_{1}\left[\begin{array}{l}
q_{2} \\
p_{2}
\end{array}\right], A_{1} A_{2}\right)
$$

The action of the element $\left(\left[\begin{array}{l}q \\ p\end{array}\right], A\right)$ on $\left[\begin{array}{l}x \\ \xi\end{array}\right]$ is defined by

$$
\left(\left[\begin{array}{l}
q \\
p
\end{array}\right], A\right)\left[\begin{array}{l}
x \\
\xi
\end{array}\right]=A\left[\begin{array}{l}
x \\
\xi
\end{array}\right]+\left[\begin{array}{l}
q \\
p
\end{array}\right]
$$

We have defined basic operations on functions and we have assigned to them corresponding transformations of the time-frequency plane. This process may be reversed: we may assign to time-frequency plane transformations corresponding operations on functions. This reversed assignment may be extended from the five generators to the whole group $\mathbb{R}^{2} \rtimes S L(2, \mathbb{R})$ just by requiring that compositions of affine maps correspond to products of operators. What comes out is a projective unitary representation $\omega$ of $\mathbb{R}^{2} \rtimes S L(2, \mathbb{R})$ on $L^{2}(\mathbb{R})$, called the extended metaplectic representation (see $[\mathbf{6}, \mathbf{9}]$ ). Summarising, the extended metaplectic representation assigns to every affine measure and orientation preserving map of the time-frequency plane a naturally corresponding unitary operator.

The Wigner distribution is a commonly used tool for representing functions on the time-frequency plane. For $f \in L^{2}(\mathbb{R})$ the Wigner distribution $W_{f}$ is defined as

$$
W_{f}(x, \xi)=\int_{-\infty}^{\infty} e^{-2 \pi i \xi p} f(x+p / 2) \overline{f(x-p / 2)} d p
$$

It was introduced in physics as a substitute for the nonexistent joint probability distribution of position and momentum. We recall its basic properties. 
Marginal properties

$$
\int_{-\infty}^{\infty} W_{f}(x, \xi) d x=|\mathcal{F} f(\xi)|^{2}, \int_{-\infty}^{\infty} W_{f}(x, \xi) d \xi=|f(x)|^{2}
$$

The Heisenberg inequality

$$
\int_{-\infty}^{\infty} \int_{-\infty}^{\infty}\left(|x-a|^{2}+|\xi-b|^{2}\right) W_{f}(x, \xi) d x d \xi \geqslant \frac{\|f\|_{L^{2}}^{2}}{2 \pi}
$$

\section{Orthogonality relations}

$$
\left\langle W_{f_{1}, g_{1}}, W_{f_{2}, g_{2}}\right\rangle=\left\langle f_{1}, f_{2}\right\rangle \overline{\left\langle g_{1}, g_{2}\right\rangle},
$$

where by $W_{f, g}$ we denote the bilinear form generated by the Wigner distribution (replace the factor $f(x-p / 2)$ by $g(x-p / 2)$ in (1.1)),

Faithfulness

$$
\text { if } W_{f}=W_{g} \text {, then } f=c g \text { for some complex } c,|c|=1,
$$

Invariance with respect to the extended metaplectic representation

$$
W_{\omega_{\tau} f}=W_{f} \circ \tau^{-1}, \text { for } \tau \in \mathbb{R}^{2} \rtimes S L(2, \mathbb{R}) .
$$

The value $W_{f}(x, \xi)$ is often interpreted as the intensity of the frequency $\xi$ at the moment $x$.

The Weyl calculus of pseudodifferential operators is another commonly used timefrequency tool. For $\sigma \in \mathcal{S}^{\prime}\left(\mathbb{R}^{2}\right)$ the formula

$$
\left\langle\sigma^{w} f, g\right\rangle=\int_{-\infty}^{\infty} \int_{-\infty}^{\infty} \sigma(x, \xi) W_{f, g}(x, \xi) d x d \xi
$$

defines an operator from the Schwartz class $\mathcal{S}(\mathbb{R})$ into the space of tempered distributions $\mathcal{S}^{\prime}(\mathbb{R})$. If $f, g \in \mathcal{S}(\mathbb{R})$, then $W_{f, g} \in \mathcal{S}\left(\mathbb{R}^{2}\right)$, and the integral in (1.6) expresses the duality between tempered distributions and Schwartz class functions. The operator $\sigma^{w}$ is called the Weyl pseudodifferential operator with symbol $\sigma$. The Weyl operator $\sigma^{w}$ with a bounded symbol $\sigma$ is interpreted in electrical engineering as the localisation operator which restricts the time-frequency content of a signal to the support of the symbol $\sigma$.

The invariance of the Wigner distribution with respect to the extended metaplectic representation (1.6) has its counterpart in terms of Weyl calculus, namely

$$
\omega_{\tau} \sigma^{w} \omega_{\tau}^{-1}=\left(\sigma \circ \tau^{-1}\right)^{w}
$$


The meaning of formulas (1.5) and (1.7) may be expressed as follows:

- the action of $\omega_{\tau}$ on $f$ corresponds to a change of variables by $\tau^{-1}$ in the time-frequency representation $W_{f}$ of $f$;

- conjugating the pseudodifferential operator $\sigma^{w}$ with $\omega_{\tau}$ corresponds to changing variables by $\tau^{-1}$ on the symbol level.

Both formulas (1.5) and (1.7) nicely fit with the interpretations mentioned before, and indicate once again that the action of the extended metaplectic representation $\omega_{r}$ may be interpreted as the transformation of the time-frequency plane given by $\tau$. For details we refer the reader to $[6,7,9,10]$.

The analysis related to the time-frequency plane splits naturally into three levels:

- the level of time-frequency plane-representing functions on the timefrequency plane as $W_{f}$;

- the operator level-studying the operators $\omega_{\tau}, \sigma^{w}$;

- the group level-formal calculus on the group $\mathbb{R}^{2} \rtimes S L(2, \mathbb{R})$.

We stress that the group $\mathbb{R}^{2} \rtimes S L(2, \mathbb{R})$ exhausts the list of all affine transformations which map Wigner distributions to Wigner distributions. Thus it is the full set of all possible affine maps of the time-frequency plane into itself. This is the reason why the group $\mathbb{R}^{2} \rtimes S L(2, \mathbb{R})$ and the extended metaplectic representation, which reflects the actions of the group elements on functions, define a natural context for investigating time-frequency phenomena.

The first problem we address is: List all possible reproducing formulas which arise in the context of the extended metaplectic representation. We restrict our attention to group related reproducing formulas, that is to formulas of the form

$$
f=\int_{H}\left\langle f, \omega_{h} \phi\right\rangle \omega_{h} \phi d h
$$

where $H$ is a connected Lie subgroup of $\mathbb{R}^{2} \rtimes S L(2, \mathbb{R})$, and $f, \phi \in L^{2}(\mathbb{R})$. We list all the connected Lie subgroups of $\mathbb{R}^{2} \times S L(2, \mathbb{R})$, we check which of them lead to reproducing formulas and we formulate explicit admissibility conditions for $\phi$ in each case. Finally, we show that the conjugacy relation properly classifies subgroups from the point of view of reproducing formulas. Our results are contained in Section 2 in Theorems 2.1, 2.2.

Reproducing formulas have a long history in the theory of function spaces and have a well established role in applications (see $[2,5]$ ). We mention that the Calderón reproducing formula is essential in Littlewood-Paley theory [8], while the reproducing formula based on the Schrödinger representation is fundamental in the theory of modulation spaces $[4,5]$. Both formulas appear in our list. Our contribution may also be 
considered as a continuation, on the group theoretic level, of the engineering program initiated in [1].

Next we show a heuristic argument, which explains why uniform coverings of the time-frequency plane are closely related to reproducing formulas. Let $\phi_{h}$ be a family of square integrable functions indexed by a parameter $h \in H, H$ a set. Let $\mu$ be a measure on $H$ and let us assume that

$$
\int_{H} W_{\phi_{h}}(x, \xi) d \mu(h)=1 \text { for all } x, \xi .
$$

Then for $f, g \in L^{2}(\mathbb{R})$ we have

$$
\begin{aligned}
\int_{H}\left\langle f, \phi_{h}\right\rangle\left\langle\phi_{h}, g\right\rangle d \mu(h) & =\int_{H}\left\langle W_{f, g}, W_{\phi_{h}}\right\rangle d \mu(h) \\
& =\left\langle W_{f, g}, \int_{H} W_{\phi_{h}} d \mu(h)\right\rangle \\
& =\int_{-\infty}^{\infty} \int_{-\infty}^{\infty} W_{f, g}(x, \xi) d x d \xi=\langle f, g\rangle .
\end{aligned}
$$

In the above argument we used the orthogonality relations (1.4) and the analogue of (1.2) for $W_{f, g}$. Clearly the chain of equalities (1.9) leads to the identity

$$
f=\int_{H}\left\langle f, \phi_{h}\right\rangle \phi_{h} d \mu(h)
$$

that is, to a reproducing formula. Condition (1.8) expresses the fact that the timefrequency plane is uniformly covered by $\phi_{h}$. Although the above argument is nice and simple, it is not formally correct. The change of order of integration in the second step of (1.9) is not allowed and the argument itself may yield false admissibility conditions. In our proofs we adapt a different approach, based on Plancherel's formula.

The second problem we address is: Find descriptions, in terms of Weyl calculus, of the commutative von Neumann algebras generated by restriction of the extended metaplectic representation to one-parameter subgroups of $\mathbb{R}^{2} \rtimes S L(2, \mathbb{R})$. A restriction of the extended metaplectic representation to a one-parameter subgroup has a nice description. Let $g_{t}$ be a one-parameter subgroup of $\mathbb{R}^{2} \rtimes S L(2, \mathbb{R})$. There is precisely one element $P$ of the algebra $\widetilde{\mathcal{Q}}$ of real polynomials of $x, \xi$ of degree at most 2, modulo constants, for which

$$
\omega_{g_{\mathrm{t}}}=e^{2 \pi i t P^{w}} .
$$

Formula (1.10) follows from the fact that the Lie algebra of $\mathbb{R}^{2} \rtimes S L(2, \mathbb{R})$ and the algebra $\tilde{\mathcal{Q}}$ equipped with the Poisson bracket are isomorphic [6]. 
At the beginning of Section 3 we observe that by conjugating with the extended metaplectic representation one may reduce any $P^{w}$ to one of the following: $P_{e}^{w}, P_{h}^{w}$, $P_{d}^{w}, P_{p}^{w}, P_{l}^{w}$, where

$$
\begin{aligned}
& P_{e}(x, \xi)=x^{2}+\xi^{2} \\
& P_{h}(x, \xi)=x \xi \\
& P_{d}(x, \xi)=\xi^{2} \\
& P_{p}(x, \xi)=\xi-x^{2} \\
& P_{l}(x, \xi)=\xi
\end{aligned}
$$

The polynomials $P_{J}, J \in \mathcal{T}=\{e, h, d, p, l\}$ correspond to nonconjugate one dimensional subgroups of $\mathbb{R}^{2} \rtimes S L(2, \mathbb{R})$.

In view of (1.10) the von Neumann algebras generated by $\omega_{g_{t}}$, where $g_{t}$ is a oneparameter subgroup of $\mathbb{R}^{2} \times S L(2, \mathbb{R})$, may be described as

$$
\mathcal{A}_{P}=\left\{M\left(P^{w}\right): M \text { is a bounded function }\right\}
$$

where $P$ is the polynomial corresponding to $g_{t}$. By conjugating with the extended metaplectic representation, one may reduce the general $\mathcal{A}_{P}$ to one of the $\mathcal{A}_{P_{J}}$. For each $J \in \mathcal{T}$ one may construct a spectral measure diagonalising $P_{J}^{w}$.

We prove that for a bounded operator $\sigma^{w}$ the following conditions are equivalent: $\sigma^{w}$ is an element of $\mathcal{A}_{P}$, the symbol $\sigma$ is constant on the level lines of $P, \sigma^{w}$ commutes with $e^{2 \pi i t P^{w}}, t \in \mathbb{R}$. In the case when the level lines of $P$ are not connected we need an extra assumption on $\sigma^{w}$, namely that $\sigma^{w}$ is a $D$-class operator with respect to the decomposition of $L^{2}(\mathbb{R})$ induced by the polynomial $P$. This result is contained in Theorem 3.1.

The operators belonging to $\mathcal{A}_{P}$ may be represented both in terms of multipliers as $M\left(P^{w}\right)$ and in terms of Weyl symbols as $\sigma^{w}$. Theorem 3.2 contains formulas which allow one to express symbols in terms of multipliers. The algebras $\mathcal{A}_{P_{e}}, \mathcal{A}_{P_{l}}$ and $\mathcal{A}_{P_{d}}$ are standard: $\mathcal{A}_{P_{e}}$ is the algebra generated by the Hermite operator, $\mathcal{A}_{P_{l}}$ is the algebra of bounded convolution operators, and $\mathcal{A}_{P_{d}}$ is a subalgebra of $\mathcal{A}_{P_{l}}$. The whole picture of operator algebras $\mathcal{A}_{P}$ expressed in terms of affine transformations of the time-frequency plane and the multiplier-symbol correspondence for $\mathcal{A}_{P_{h}}, \mathcal{A}_{P_{p}}$, however, seem to be new.

The operators $M\left(P^{w}\right)$ have a nice interpretation. For $M=\chi_{[a, b]}$ they are interpreted as localisation operators restricting time-frequency content of functions to the domain $a \leqslant P(x, \xi) \leqslant b$. 


\section{REPRODUCING FORMULAS}

Let $H$ be a locally compact group, $d h$ its left Haar measure, and $\omega$ a strongly continuous unitary representation of $H$ on a Hilbert space $\mathcal{H}$. A vector $\phi \in \mathcal{H}$ is called $\omega$-admissible if for all $f \in \mathcal{H}$ the reproducing formula

$$
f=\int_{H}\left\langle f, \omega_{h} \phi\right\rangle \omega_{h} \phi d h
$$

holds. The integral is understood in the weak sense. It is easy to observe that a vector $\phi \in \mathcal{H}$ is $\omega$-admissible if and only if for all $f \in \mathcal{H}$

$$
\|f\|^{2}=\int_{H}\left|\left\langle f, \omega_{h} \phi\right\rangle\right|^{2} d h .
$$

As we mentioned in the introduction, our target is to investigate and classify those reproducing formulas that come from restrictions of the extended metaplectic representation to connected Lie subgroups of the semidirect product $G=\mathbb{R}^{2} \rtimes S L(2, \mathbb{R})$.

A subgroup $H$ of $G$ is called reproducing if the set of $\omega_{\mid H}$-admissible vectors is nonempty. The admissible vectors are called $H$-admissible in this case. Observe that if two subgroups $H_{1}, H_{2}$ are conjugate, that is, $H_{1}=g^{-1} H_{2} g$ for some $g \in G$, then $H_{1}$, $H_{2}$ are either both reproducing or both nonreproducing. Moreover $\phi$ is $H_{1}$ admissible if and only if $\omega_{g} \phi$ is $H_{2}$ admissible. To see this it is enough to change variables in the integral

$$
f=\int_{H_{1}}\left\langle f, \omega_{h_{1}} \phi\right\rangle \omega_{h_{1}} \phi d h_{1}
$$

substituting $h_{1}=g^{-1} h_{2} g$.

We list now, up to conjugacy, all connected Lie subgroups of G. To this end, let

$$
d_{s}=\left[\begin{array}{cc}
s^{-1} & 0 \\
0 & s
\end{array}\right], s>0, \quad l_{t}=\left[\begin{array}{ll}
1 & 0 \\
t & 1
\end{array}\right], t \in \mathbb{R}, \quad k_{\theta}=\left[\begin{array}{cc}
\cos \theta & -\sin \theta \\
\sin \theta & \cos \theta
\end{array}\right], \theta \in \mathbb{R}
$$

Lemma 2.1. [3] Any connected Lie subgroup of $G$ is conjugate to one of the following non-conjugate subgroups. For each group we indicate the dimension, a parametrisation of the elements, with parameters $q, p, t, \theta \in \mathbb{R}$ and $s>0$, and the corresponding Haar measure:

$$
\begin{aligned}
& \left(\left[\begin{array}{l}
q \\
0
\end{array}\right], I\right), d q \\
& \left(\left[\begin{array}{l}
0 \\
0
\end{array}\right], l_{t}\right), d t
\end{aligned}
$$




$$
\begin{aligned}
& \left(\left[\begin{array}{l}
0 \\
0
\end{array}\right], d_{s}\right), \quad \frac{d s}{s} \\
& \left(\left[\begin{array}{l}
0 \\
0
\end{array}\right], k_{\theta}\right), \quad \frac{d \theta}{2 \pi} \\
& \left(\left[\begin{array}{c}
t \\
\frac{t^{2}}{2}
\end{array}\right], l_{t}\right), d t \\
& \left(\left[\begin{array}{l}
q \\
p
\end{array}\right], I\right), \quad d q d p \\
& \left(\left[\begin{array}{l}
0 \\
0
\end{array}\right], l_{t} d_{s^{2 / 2}}\right), \frac{d t d s}{s^{2}} \\
& \left(\left[\begin{array}{l}
0 \\
p
\end{array}\right], d_{s}\right), \frac{d p d s}{s^{2}} \\
& \left(\left[\begin{array}{l}
0 \\
p
\end{array}\right], l_{t}\right), \quad d p d t \\
& \left(\left[\begin{array}{l}
t \\
p
\end{array}\right], l_{t}\right), \quad d p d t \\
& \left(\left[\begin{array}{l}
0 \\
0
\end{array}\right], l_{t} d_{s^{1 / 2}} k_{\theta}\right), \frac{d t d s d \theta}{2 \pi s^{2}} \\
& \left(\left[\begin{array}{l}
0 \\
p
\end{array}\right], l_{t} d_{s^{1 / 2}}\right), \frac{d p d t d s}{s^{5 / 2}} \\
& \left(\left[\begin{array}{l}
q \\
p
\end{array}\right], l_{t}\right), \quad d q d p d t \\
& \left(\left[\begin{array}{l}
q \\
p
\end{array}\right], d_{s}\right), \quad \frac{d q d p d s}{s} \\
& \left(\left[\begin{array}{l}
q \\
p
\end{array}\right], k_{\theta}\right), \quad \frac{d q d p d \theta}{2 \pi} ; \\
& \left(\left[\begin{array}{l}
q \\
p
\end{array}\right], l_{t} d_{s^{1 / 2}}\right), \frac{d q d p d t d s}{s^{2}} \\
& \left(\left[\begin{array}{l}
q \\
p
\end{array}\right], l_{t} d_{s^{1 / 2}} k_{\theta}\right), \frac{d q d p d t d s d \theta}{2 \pi s^{2}} \text {. }
\end{aligned}
$$

THEOREM 2.1. The only connected reproducing Lie subgroups of $G$ are (2.i), (2.ii), (2.iii), (2.v), (3.v) and their conjugates. The corresponding admissibility conditions are:

$$
\|\phi\|_{L^{2}}=1
$$




$$
\int_{0}^{\infty}|\phi(x)|^{2} \frac{d x}{x^{2}}=\int_{0}^{\infty}|\phi(-x)|^{2} \frac{d x}{x^{2}}=\frac{1}{2}, \int_{0}^{\infty} \phi(x) \overline{\phi(-x)} \frac{d x}{x^{2}}=0
$$

$$
\int_{0}^{\infty}|\phi(x)|^{2} \frac{d x}{x}=\int_{0}^{\infty}|\phi(-x)|^{2} \frac{d x}{x}=1
$$

$$
\begin{gathered}
\|\phi\|_{L^{2}}=1 \\
\|\phi\|_{L^{2}}=1 .
\end{gathered}
$$

Proof: We first show the positive results and then the negative ones.

Positive Results. Cases (2.i), (2.iii) are standard and we do not include their proofs.

CASE (2.ii). Since

$$
\omega_{l_{t} d_{s} 1 / 2} \phi(x)=e^{\pi i t x^{2}} s^{1 / 4} \phi\left(s^{1 / 2} x\right)
$$

and for $F \in L^{2}(\mathbb{R})$

$$
\int_{-\infty}^{\infty}\left|\int_{-\infty}^{\infty} F(x) e^{-\pi i t x^{2}} d x\right|^{2} d t=\int_{0}^{\infty} \frac{|F(x)+F(-x)|^{2}}{x} d x
$$

we obtain

$$
\begin{aligned}
& \int_{H_{(2 . i i)}}\left|\left\langle f, \omega_{h} \phi\right\rangle\right|^{2} d h \\
& =\int_{0}^{\infty} \int_{-\infty}^{\infty}\left|\int_{-\infty}^{\infty} f(x) s^{1 / 4} \bar{\phi}\left(s^{1 / 2} x\right) e^{-\pi i t x^{2}}\right|^{2} d t \frac{d s}{s^{2}} \\
& =\int_{0}^{\infty} \int_{0}^{\infty}\left\{|f(x)|^{2} s^{1 / 2}\left|\phi\left(s^{1 / 2} x\right)\right|^{2}+|f(-x)|^{2} s^{1 / 2}\left|\phi\left(-s^{1 / 2} x\right)\right|^{2}\right. \\
& \left.\quad+2 \operatorname{Re} f(x) \bar{f}(-x) s^{1 / 2} \bar{\phi}\left(s^{1 / 2} x\right) \phi\left(-s^{1 / 2} x\right)\right\} \frac{d x}{x} \frac{d s}{s^{2}}
\end{aligned}
$$

for $\phi \in L^{2}(\mathbb{R})$ and $f$ bounded, with support contained in $\{x \in \mathbb{R}: r \leqslant|x| \leqslant R\}$, $0<r<R<\infty$. Case (2.ii) follows easily from (2.4).

CASE (2.v). Since

$$
\omega\left(\left[\begin{array}{l}
t \\
p
\end{array}\right], l_{t}\right) \phi(x)=e^{2 \pi i p x} e^{\pi i t(x-t)^{2}} \phi(x-t),
$$

for $\phi, f \in L^{2}(\mathbb{R})$ we have

$$
\begin{aligned}
\int_{H_{(2 . v)}}\left|\left\langle f, \omega_{h} \phi\right\rangle\right|^{2} d h & =\int_{-\infty}^{\infty} \int_{-\infty}^{\infty}\left|\int_{-\infty}^{\infty} f(x) \bar{\phi}(x-t) e^{-\pi i t(x-t)^{2}} e^{-2 \pi i p x} d x\right|^{2} d p d t \\
& =\int_{-\infty}^{\infty} \int_{-\infty}^{\infty}|f(x)|^{2}|\phi(x-t)|^{2} d x d t=\|f\|_{L^{2}}^{2}\|\phi\|_{L^{2}}^{2}
\end{aligned}
$$


CASE (3.v). Observe that

$$
\omega\left(\left[\begin{array}{l}
q \\
p
\end{array}\right], k_{\theta}\right) \phi(x)=e^{2 \pi i p x} \omega_{k_{\theta}} \phi(x-q) .
$$

By case (2.i) we obtain

$$
\int_{H_{(3, v)}}\left|\left\langle f, \omega_{h} \phi\right\rangle\right|^{2} d h=\frac{1}{2 \pi} \int_{0}^{2 \pi}\|f\|_{L^{2}}^{2}\left\|\omega_{k_{\theta}} \phi\right\|_{L^{2}}^{2}=\|f\|_{L^{2}}^{2}\|\phi\|_{L^{2}}^{2} .
$$

NEGATIVE RESULTS: In each remaining case we show that formula (2.2) fails, hence (2.1) fails as well. One dimensional results are relatively straightforward, so we include only the proof of case (1.iv).

CASE (1.iv). Let $\phi=\sum_{m=0}^{\infty} \lambda_{m} e_{m}$ be the expansion of $\phi$ in terms of the Hermite system defined in (3.1). Since

$$
\omega_{k_{\theta}} \phi=\sum_{m} e^{i m \theta} \lambda_{m} e_{m}
$$

(see for example [6]), for $f \in L^{2}(\mathbb{R})$ we obtain

$$
\begin{aligned}
\int_{H_{(1 . i v)}}\left|\left\langle f, \omega_{h} \phi\right\rangle\right|^{2} d h & =\frac{1}{2 \pi} \int_{0}^{2 \pi}\left|\sum_{m} \gamma_{m} \bar{\lambda}_{m} e^{-i m \theta}\right|^{2} d \theta \\
& =\sum_{m}\left|\gamma_{m}\right|^{2}\left|\lambda_{m}\right|^{2},
\end{aligned}
$$

where $\gamma_{m}=\left\langle f, e_{m}\right\rangle$. By (2.6), no $\phi \in L^{2}(\mathbb{R})$ satisfies condition (2.2).

CASE (2.iv). Since

$$
\omega\left(\left[\begin{array}{l}
0 \\
p
\end{array}\right], l_{t}\right) \phi(x)=e^{2 \pi i p x} e^{\pi i t x^{2}} \phi(x)
$$

by Plancherel

$$
\int_{H_{(2 . i v)}}\left|\left\langle f, \omega_{h} \phi\right\rangle\right|^{2} d h=\int_{-\infty}^{\infty} \int_{-\infty}^{\infty}|f(p)|^{2}|\phi(p)|^{2} d p d t .
$$

The last integral in (2.7) either diverges or vanishes.

CASE (3.i). Let $\phi=\sum_{m=0}^{\infty} \lambda_{m} e_{m}$ be the expansion of $\phi$ in terms of the Hermite system. Clearly

$$
\omega_{l_{t} d^{1 / 2}{ }^{1} k_{\theta}} \phi(x)=e^{\pi i t x^{2}} s^{1 / 4} \omega_{k_{\theta}} \phi\left(s^{1 / 2} x\right)
$$


An application of (2.5), Parseval's formula, (2.3) and (2.8) leads to

$$
\begin{aligned}
\int_{H_{(3, i)}}\left|\left\langle f, \omega_{h} \phi\right\rangle\right|^{2} d h= & 2 \sum_{m}\left|\lambda_{m}\right|^{2}\left\{\int_{0}^{\infty}|f(x)|^{2} d x \int_{0}^{\infty}\left|e_{m}(s)\right|^{2} \frac{d s}{s^{2}}\right. \\
& +\int_{0}^{\infty}|f(-x)|^{2} d x \int_{0}^{\infty}\left|e_{m}(-s)\right|^{2} \frac{d s}{s^{2}} \\
& \left.+\int_{0}^{\infty} f(x) \bar{f}(-x) d x \int_{0}^{\infty} \bar{e}_{m}(s) e_{m}(-s) \frac{d s}{s^{2}}\right\}
\end{aligned}
$$

for $f$ bounded with support contained in $\{x \in \mathbb{R}: r \leqslant|x| \leqslant R\}, 0<r<R<\infty$. Observe that (2.9) implies $\lambda_{2 m}=0$, for $m=0,1,2, \ldots$ Indeed,

$$
\int_{0}^{\infty}\left|e_{2 m}(s)\right|^{2} \frac{d s}{s^{2}}=\infty
$$

because $e_{2 m}(0) \neq 0$. Formula (2.9) implies also that

$$
\sum_{m}\left|\lambda_{2 m+1}\right|^{2} \int_{0}^{\infty} e_{2 m+1}(s) e_{2 m+1}(-s) \frac{d s}{s^{2}}=0 .
$$

Since the functions $e_{2 m+1}$ are odd, it follows from (2.10) that $\lambda_{2 m+1}=0$, for $m=$ $0,1,2, \ldots$ This finishes the proof of case (3.i).

The remaining cases (3.ii), (3.iii), (3.iv), (4.i), (5.i) are straightforward consequences of Plancherel's theorem. We omit their proofs.

Now we classify the reproducing subgroups of $G$ taking the reproducing formulas themselves as criteria for classification. We show that two subgroups generate reproducing formulas differing by an affine transformation of the time-frequency plane if and only if they are conjugate.

Let $H_{1}, H_{2}$ be two connected Lie subgroups of $G$. We say that $H_{2}$ is equivalent to $H_{1}$ if there is a group isomorphism $\Phi: H_{1} \rightarrow H_{2}$ such that for every $H_{1}$-admissible $\phi$ there is $\psi \in L^{2}$ such that for all $f \in L^{2}$

$$
\left\langle f, \omega_{h} \phi\right\rangle \omega_{h} \phi=\left\langle f, \omega_{\Phi(h)} \psi\right\rangle \omega_{\Phi(h)} \psi
$$

This condition simply means that after a change of variables given by $\Phi$ the coefficients in the reproducing formulas are the same. It is easy to see that if $\mathrm{H}_{2}$ is equivalent to $H_{1}$ then $\psi=\phi$ modulo a phase factor, $H_{2}=H_{1}$ and $\Phi=i d$. Indeed, if $H_{2}$ is equivalent to $H_{1}$ then by putting $h=e$ in (2.11) we see that $\psi=\phi$ modulo a phase factor. It follows that for all admissible $\phi$ one has $\omega_{h} \phi=\omega_{\Phi(h)} \phi$ modulo a phase factor and this implies $\Phi(h)=h$. 
Let $g \in G$. We say that $H_{2}$ is $g$-equivalent to $H_{1}$ if there is a group isomorphism $\Phi: H_{1} \rightarrow H_{2}$ such that for every $H_{1}$-admissible $\phi$ there is $\psi \in L^{2}$ such that for all $f \in L^{2}$

$$
\left\langle f, \omega_{h} \phi\right\rangle \omega_{h} \phi=\left\langle\omega_{g} f, \omega_{\Phi(h)} \psi\right\rangle \omega_{g^{-1}} \omega_{\Phi(h)} \psi .
$$

The above condition means that after transforming the time-frequency plane by $g$ the reproducing formulas become equivalent. A similar argument as before shows that if $H_{2}$ is $g$-equivalent to $H_{1}$, then $\psi=\omega_{g} \phi$ modulo a phase factor and $\Phi(h)=g h g^{-1}$. By combining this with the previous discussion about conjugate subgroups, we obtain

THEOREM 2.2. Let $H_{1}, H_{2}$ be connected Lie subgroups of $G$ and let $g \in G$. The subgroup $H_{2}$ is $g$-equivalent to $H_{1}$ if and only if $H_{1}=g^{-1} H_{2} g$.

\section{Comments.}

(i) It follows easily from our proof that the group $S L(2, \mathbb{R})$ (case (3.i) in our notation) provides reproducing formulas on $L^{2}\left(\mathbb{R}_{+}\right)$and $L^{2}\left(\mathbb{R}_{-}\right)$.

(ii) The class of distinct subgroups which are conjugate to a given subgroup $H$ of $G$ may be described in terms of the normaliser $N(H)$ of $H$ in $G$, since it is in bijective correspondence with the quotient $G / N(H)$. On the other hand, we know that conjugacy corresponds to equivalence with respect to reproducing formulas. The form of $N(H)$ for the subgroups $H$ of $\mathbb{R}^{2} \rtimes S L(2, \mathbb{R})$ listed in Theorem (2.1) (which form a complete set of representatives under conjugacy) are given in [3].

\section{Commutative Operator Algebras $\mathcal{A}_{P}$}

Let $\mathcal{Q}$ denote the algebra of real polynomials of $x, \xi$ of degree at most 2. For $P \in \mathcal{Q}$, let $P^{w}$ denote the Weyl pseudodifferential operator defined by the symbol $P$. The operator $P^{w}$ maps the Schwartz class $\mathcal{S}(\mathbb{R})$ continuously into itself and it extends to a continuous operator on the space of tempered distributions $\mathcal{S}(\mathbb{R})$. As we have indicated in the introduction, we want to represent, in terms of Weyl calculus, the commutative von Neumann algebras $\mathcal{A}_{P}=\left\{M\left(P^{w}\right): M\right.$ bounded $\}$ and describe the correspondence between multiplier and symbol representations. Recall that by $G$ we denote the group $R^{2} \rtimes S L(2, \mathbb{R})$ and that for $\tau \in G$

$$
\omega_{\tau} P^{w} \omega_{\tau}^{*}=\left(P \circ \tau^{-1}\right)^{w}
$$

The general strategy, in order to achieve the desired descriptions, will be to use the above formula and reduce the problem to the case of the five main algebras corresponding to the polynomials defined in (1.11). The following proposition shows that for any $P \in \mathcal{Q}$ one may choose an appropriate change of coordinates $\tau \in G$ and bring $P$ to 
one of the five forms $P_{J}, J \in \mathcal{T}=\{e, h, d, p, l\}$. On the level of operators this means that by conjugating with the extended metaplectic representation one may reduce any $P^{w}$ to one of the $P_{J}^{w}$.

Proposition 3.1. For any $P \in \mathcal{Q}$ there are $\tau \in G, J \in \mathcal{T}$ and $\lambda, c \in \mathbb{R}$ such that

$$
P(x, \xi)=\lambda P_{J}\left(\tau^{-1}(x, \xi)\right)+c .
$$

Proof: The proof follows by an easy computation and we omit it.

It is easy to check that the explicit forms of the operators $P_{J}^{w}$ are:

$$
\begin{aligned}
& P_{e}^{w}=X^{2}+D^{2}, \\
& P_{h}^{w}=\frac{X D+D X}{2}, \\
& P_{d}^{w}=D^{2}, \\
& P_{p}^{w}=D-X^{2}, \\
& P_{l}^{w}=D,
\end{aligned}
$$

where $X f(x)=x f(x), D f(x)=(1 / 2 \pi i) f^{\prime}(x)$.

Next we introduce the following systems of functions:

$$
\begin{aligned}
& e_{m}(x)=\frac{2^{1 / 4}}{(m !)^{1 / 2}}\left(\frac{-1}{2 \pi^{1 / 2}}\right)^{m} e^{\pi x^{2}} \frac{d^{m}}{d x^{m}}\left(e^{-2 \pi x^{2}}\right), m=0,1, \ldots, \\
& l_{\lambda}(x)=e^{2 \pi i \lambda x}, \lambda \in \mathbb{R}, \\
& h_{\lambda}(x)=(\lambda x)^{-1 / 2} e^{2 \pi i \log \lambda \log x}, \lambda>0 \\
& p_{\lambda}(x)=e^{2 \pi i\left(\lambda x+\left(x^{3} / 3\right)\right)}, \lambda \in \mathbb{R} .
\end{aligned}
$$

Clearly, all systems are defined on $\mathbb{R}$ except (3.3) which is defined on the positive half line. System (3.1) gives an orthonormal basis of $L^{2}(\mathbb{R})$, called the Hermite basis. It is well known that it diagonalises the Hermite operator $P_{e}^{w}$, and that

$$
P_{e}^{w} e_{m}=\frac{m+1 / 2}{\pi} e_{m}
$$

System (3.2) is also standard. It diagonalises $P_{l}^{w}$,

$$
P_{l}^{w} l_{\lambda}=\lambda l_{\lambda}
$$

induces the spectral measure $E_{l}$

$$
E_{l}(A) f=\int_{A}\left\langle f, l_{\lambda}\right\rangle l_{\lambda} d \lambda, A \subset \mathbb{R}
$$


and defines a selfadjoint extension $T_{l}$ of $P_{l}^{w}$, namely

$$
T_{l}=\int_{-\infty}^{\infty} \lambda d E_{l}(\lambda)
$$

The symbol calculus for $P_{l}^{w}$ is defined as follows: for $M \in L^{\infty}(\mathbb{R})$

$$
M\left(T_{l}\right)=\int M(\lambda) d E_{l}(\lambda)=C_{M}
$$

where $C_{M}$ is the operator of convolution with the inverse Fourier transform of $M$. Systems (3.3), (3.4) play similar roles for $P_{h}^{w}, P_{p}^{w}$ as (3.2) for $P_{l}^{w}$, as illustrated below.

Proposition 3.2 .

$$
P_{h}^{w} h_{\lambda}=\log \lambda h_{\lambda}, \quad P_{p}^{w} p_{\lambda}=\lambda p_{\lambda} .
$$

PROOF: The proof follows easily by direct differentiation.

Proposition 3.3. The formulas

$$
\mathcal{F}_{h} f(\lambda)=\int_{0}^{\infty} f(x) \overline{h_{\lambda}(x)} d x, \quad \mathcal{F}_{p} f(\lambda)=\int_{-\infty}^{\infty} f(x) \overline{p_{\lambda}(x)} d x
$$

define unitary maps $\mathcal{F}_{h}: L^{2}\left(\mathbb{R}_{+}\right) \rightarrow L^{2}\left(\mathbb{R}_{+}\right), \mathcal{F}_{p}: L^{2}(\mathbb{R}) \rightarrow L^{2}(\mathbb{R})$ which satisfy the relations

$$
W_{h} \mathcal{F}_{h}=\mathcal{F} W_{h}, \quad \mathcal{F}_{p}=\mathcal{F} W_{p},
$$

where $W_{h}: L^{2}\left(\mathbb{R}_{+}\right) \rightarrow L^{2}(\mathbb{R})$ is defined by $W_{h} f(w)=f\left(e^{w}\right) e^{w / 2}$, and $W_{p}: L^{2}(\mathbb{R}) \rightarrow$ $L^{2}(\mathbb{R})$ is defined by $W_{p} f(x)=f(x) e^{-2 \pi i\left(x^{3} / 3\right)}$.

PROOF: The proof follows directly from Plancherel's theorem.

Proposition 3.4. (i) The formulas

$$
E_{h}(A) f=\int_{A}\left\langle f, h_{\lambda}\right\rangle h_{\lambda} d \lambda, A \subset \mathbb{R}_{+}, \quad E_{p}(B) g=\int_{B}\left\langle g, p_{\lambda}\right\rangle p_{\lambda} d \lambda, B \subset \mathbb{R},
$$

define spectral measures on $L^{2}\left(\mathbb{R}_{+}\right)$and $L^{2}(\mathbb{R})$, respectively. After conjugating with $W_{h}$ and $W_{p}$, the spectral measures $E_{h}$ and $E_{p}$ reduce to $E_{l}$,

$$
W_{h} E_{h}(A) W_{h}^{-1}=E_{l}(\log A), \text { and } \quad W_{p} E_{p}(B) W_{p}^{-1}=E_{l}(B) .
$$

(ii) The operators

$$
\widetilde{T}_{h}=\int_{0}^{\infty} \log \lambda d E_{h}(\lambda), \quad T_{p}=\int_{-\infty}^{\infty} \lambda d E_{p}(\lambda)
$$


with domains

$$
\begin{aligned}
D\left(\tilde{T}_{h}\right) & =\left\{f \in L^{2}\left(\mathbb{R}_{+}\right): \int_{0}^{\infty}(\log \lambda)^{2}\left|\left\langle f, h_{\lambda}\right\rangle\right|^{2} d \lambda<\infty\right\}, \\
D\left(T_{p}\right) & =\left\{g \in L^{2}(\mathbb{R}): \int_{-\infty}^{\infty} \lambda^{2}\left|\left\langle g, p_{\lambda}\right\rangle\right|^{2} d \lambda<\infty\right\}
\end{aligned}
$$

are selfadjoint extensions of $P_{h}^{w}, P_{p}^{w} ;$ for $f \in C_{c}^{\infty}\left(\mathbb{R}_{+}\right), g \in C_{c}^{\infty}(\mathbb{R})$

$$
\tilde{T}_{h} f=P_{h}^{w} f, \quad T_{p} g=P_{p}^{w} g .
$$

(iii) Let $M \in L^{\infty}(\mathbb{R})$. After conjugating with $W_{h}$ and $W_{p}$, respectively, the operators

$$
M\left(\tilde{T}_{h}\right)=\int_{0}^{\infty} M(\log \lambda) d E_{h}(\lambda), \quad M\left(T_{p}\right)=\int_{-\infty}^{\infty} M(\lambda) d E_{p}(\lambda)
$$

reduce to standard convolution operators, namely

$$
W_{h} M\left(\tilde{T}_{h}\right) W_{h}^{-1}=C_{M}, \quad W_{p} M\left(T_{p}\right) W_{p}^{-1}=C_{M} .
$$

Proof: The proof follows easily from Propositions 3.2, 3.3 and standard properties of spectral measures.

In the above proposition we have defined $\widetilde{T}_{h}$ and $T_{p}$, the selfadjoint extensions of $P_{h \mid C_{c}^{\infty}}^{w}$ and $P_{p}^{w}$. We extend the operator $\tilde{T}_{h}$ to $T_{h}$, from the half line, to the whole line, by symmetry. By $T_{e}, T_{l}, T_{d}$ we denote the selfadjoint extensions of $P_{e}^{w}$, $P_{l}^{w}, P_{d}^{w}$ defined in terms of the corresponding spectral measures. The unitary groups $U_{t}^{e}=e^{\pi i t T_{e}}, U_{t}^{h}=e^{2 \pi i t T_{h}} U_{t}^{l}=e^{2 \pi i t T_{l}}, U_{t}^{d}=e^{2 \pi i t T_{d}}$ are very well known. They are: the Hermite group

$$
U_{t}^{e} f=\sin ^{-1 / 2} t \int_{-\infty}^{\infty} e^{-\pi i(\cot t)\left(\cdot^{2}+y^{2}\right)+2 \pi i(\cdot y / \sin t)} f(y) d y
$$

the dilation group

$$
U_{t}^{h} f=e^{t / 2} f\left(e^{t} \cdot\right)
$$

the translation group

$$
U_{t}^{l} f=f(\cdot+t)
$$

and the group of convolutions with imaginary gaussians

$$
U_{t}^{d} f=\mathcal{F}^{-1} e^{2 \pi i t^{2}} \mathcal{F}
$$

Finally, the group $U_{t}^{p}=e^{2 \pi i t T_{p}}$ has the form $U_{t}^{p} f=e^{-2 \pi i\left(t .{ }^{2}+t^{2}\right)} f(\cdot+t)$.

As in the case of the extended metaplectic representation we identify unitary operators which differ by multiplicative constants.

The standard fact that the operators commuting with translations are convolution operators, has its counterparts for $\widetilde{U}_{t}^{h}=e^{2 \pi i t \widetilde{T}_{h}}$ and $U_{t}^{p}$. 


\section{PROPOSITION 3.5.}

(i) A bounded operator $\widetilde{T}$ defined on $L^{2}\left(\mathbb{R}_{+}\right)$commutes with $\widetilde{U}_{t}^{h}$, for all $t \epsilon$ $\mathbb{R}$, if and only if there is a function $M \in L^{\infty}(\mathbb{R})$ such that $\widetilde{T}=M\left(\widetilde{T_{h}}\right)$,

(ii) $A$ bounded operator $T$ defined on $L^{2}(\mathbb{R})$ commutes with $U_{t}^{p}$, for all $t \in \mathbb{R}$, if and only if there is a function $M \in L^{\infty}(\mathbb{R})$ such that $T=M\left(T_{p}\right)$.

PROOF: The proof follows by an adaptation of the standard argument.

Let now $U_{t}^{P}$ and $g_{t}^{P}$ denote the unitary group and the one-parameter subgroup of $G$ corresponding to the polynomial $P \in \mathcal{Q}$, respectively, and let $\sigma \in \mathcal{S}^{\prime}\left(\mathbb{R}^{2}\right)$. We want to describe the geometric conditions on the symbol $\sigma$ that reflect the commutativity property $U_{t}^{P} \sigma^{w} U_{-t}^{P}=\sigma^{w}$.

PRoposition 3.6. Let $\sigma \in \mathcal{S}^{\prime}\left(\mathbb{R}^{2}\right)$ and let $f, g \in \mathcal{S}(\mathbb{R})$. Then

$$
\left\langle U_{t}^{P} \sigma^{w} U_{-t}^{P} f, g\right\rangle=\left\langle\left(\sigma \circ g_{-t}^{P}\right)^{w} f, g\right\rangle \text {. }
$$

Proof: This is a special case of (1.7).

Let us look again at the five basic polynomials. Recall that, as we know from (1.10), the unitary groups $U_{t}^{J}, J \in \mathcal{T}$, are the restrictions of the extended metaplectic representation to the one-parameter subgroups $g_{t}^{J}$. The explicit forms of $g_{t}^{J}$ are:

$$
\begin{aligned}
g_{t}^{e}\left[\begin{array}{l}
x \\
\xi
\end{array}\right] & =\left[\begin{array}{cc}
\cos t & -\sin t \\
\sin t & \cos t
\end{array}\right]\left[\begin{array}{l}
x \\
\xi
\end{array}\right] \\
g_{t}^{h}\left[\begin{array}{l}
x \\
\xi
\end{array}\right] & =\left[\begin{array}{cc}
e^{-t} & 0 \\
0 & e^{t}
\end{array}\right]\left[\begin{array}{l}
x \\
\xi
\end{array}\right] \\
g_{t}^{d}\left[\begin{array}{l}
x \\
\xi
\end{array}\right] & =\left[\begin{array}{cc}
1 & -t \\
0 & 1
\end{array}\right]\left[\begin{array}{l}
x \\
\xi
\end{array}\right] \\
g_{t}^{p}\left[\begin{array}{l}
x \\
\xi
\end{array}\right] & =\left[\begin{array}{c}
x-t \\
\xi-x^{2}+(x-t)^{2}
\end{array}\right] \\
g_{t}^{l}\left[\begin{array}{l}
x \\
\xi
\end{array}\right] & =\left[\begin{array}{c}
x-t \\
\xi
\end{array}\right] .
\end{aligned}
$$

The orbits of $g_{t}^{J}$ parametrise the connected components of the level lines of $P_{J}$. By Proposition 3.1, this fact holds in general and we are led to the following definition.

Let $P \in \mathcal{Q}$ and let $\sigma \in \mathcal{S}^{\prime}\left(\mathbb{R}^{2}\right)$. We say that the distribution $\sigma$ is constant on the level lines of $P$ if:

(i) in the case of connected level lines of $P$ (e,p,l and conjugate cases): $\sigma \circ g_{t}^{P}$ does not depend on $t$,

(ii) in the case of non-connected level lines of $P$ ( $h, d$ and conjugate cases): $\sigma \circ g_{t}^{P}$ does not depend on $t$ and $\sigma \circ \tau$ is even, $\tau$ is an affine transformation bringing $P$ to the form $P_{h}$ or $P_{d}$. 
We stress that in case (ii) the level lines have two components, and the orbits parametrise only one component. This is why one needs an extra symmetry condition.

Next we define decompositions of $L^{2}(\mathbb{R})$ and D-class operators corresponding to $P \in \mathcal{Q}$. Since both these notions reflect the structure of level lines of $P$, we present them together. We do it first for $P_{J}, J \in \mathcal{T}$, and then, by conjugation, for all $P \in \mathcal{Q}$.

There are two types of level lines of $P_{h}$ : the connected line $x \xi=0$, and the non-connected lines $x \xi=c, c \neq 0$. The set $\mathbb{R}^{2} \backslash\{(x, \xi): x \xi=0\}$ has four components:

$$
\begin{aligned}
I & =\{(x, \xi): x, \xi>0\}, \quad I I=\{(x, \xi): x<0, \xi>0\}, \\
I I I & =\{(x, \xi): x, \xi<0\}, \quad I V=\{(x, \xi): x>0, \xi<0\} .
\end{aligned}
$$

The direct sum

$$
L^{2}(\mathbb{R})=\mathcal{H}_{I} \oplus \mathcal{H}_{I I} \oplus \mathcal{H}_{I I I} \oplus \mathcal{H}_{I V}
$$

where

$$
\begin{aligned}
\mathcal{H}_{I} & =\left\{f \in L^{2}\left(\mathbb{R}_{+}\right): \mathcal{F}_{h} f(\lambda)=0 \text { for } \lambda>1\right\}, \\
\mathcal{H}_{I V} & =\left\{f \in L^{2}\left(\mathbb{R}_{+}\right): \mathcal{F}_{h} f(\lambda)=0 \text { for } \lambda<1\right\}, \\
\mathcal{H}_{I I} & =\left\{f(-\cdot): f \in \mathcal{H}_{I V}\right\}, \quad \mathcal{H}_{I I I}=\left\{f(-\cdot): f \in \mathcal{H}_{I}\right\},
\end{aligned}
$$

corresponds to the decomposition $\mathbb{R}^{2} \backslash\{(x, \xi): x \xi=0\}=I \cup I I \cup I I I \cup I V$, and is called the decomposition of $L^{2}(\mathbb{R})$ induced by $P_{h}$ (for an intuitive explanation of this correspondence see the comments following this section). We say that a bounded operator $T$ acting on $L^{2}(\mathbb{R})$ is D-class with respect to the decomposition induced by $P_{h}$ if $\mathcal{H}_{K}$ is an invariant subspace of $T$ for $K=I, I I, I I I, I V$ and

$$
T_{\mid \mathcal{H}_{I I I}}=V^{-1} T_{\mid \mathcal{H}_{I}} V, T_{\mid \mathcal{H}_{I I}}=V^{-1} T_{\mid \mathcal{H}_{I V}} V
$$

where $V f(x)=f(-x)$.

The polynomial $P_{d}$ also has connected and non-connected level lines. After removing the connected level line we get the two components:

$$
I=\{(x, \xi): \xi>0\}, I I=\{(x, \xi): \xi<0\} .
$$

This decomposition induces the direct sum

$$
L^{2}(\mathbb{R})=\mathcal{D}_{I} \oplus \mathcal{D}_{I I},
$$

where

$$
\begin{aligned}
\mathcal{D}_{I} & =\left\{f \in L^{2}(\mathbb{R}): \mathcal{F} f(\lambda)=0, \lambda>0\right\} \\
\mathcal{D}_{I I} & =\left\{f \in L^{2}(\mathbb{R}): \mathcal{F} f(\lambda)=0, \lambda<0\right\} .
\end{aligned}
$$


A bounded operator $T$ is called $D$-class with respect to the decomposition induced by $P_{d}$ if $\mathcal{D}_{I}, \mathcal{D}_{I I}$ are its invariant spaces and

$$
T_{\mid \mathcal{D}_{I I}}=V^{-1} T_{\mid \mathcal{D}_{I}} V
$$

The polynomials $P_{e}, P_{p}, P_{l}$ have only connected level lines and they induce the trivial decomposition, just $L^{2}(\mathbb{R})$.

Finally, let $P \in \mathcal{Q}$ be arbitrary and let $\tau \in G$ be an affine transformation which brings $P$ to one of the forms $P_{J}, J \in \mathcal{T}$.

If $J=h$ we define the decomposition of $L^{2}(\mathbb{R})$ induced by $P$ as

$$
\omega_{\tau} \mathcal{H}_{I} \oplus \omega_{\tau} \mathcal{H}_{I I} \oplus \omega_{\tau} \mathcal{H}_{I I I} \oplus \omega_{\tau} \mathcal{H}_{I V}
$$

and a bounded operator $T$ is called D-class if $\omega_{\tau}^{-1} T \omega_{\tau}$ is D-class with respect to $P_{h}$.

If $J=d$ the decomposition is defined as

$$
\omega_{\tau} \mathcal{D}_{I} \oplus \omega_{\tau} \mathcal{D}_{I I}
$$

and a bounded operator is called D-class if $\omega_{\tau}^{-1} T \omega_{\tau}$ is D-class with respect to $P_{d}$.

In the other cases the decomposition is trivial and the D-class condition is void.

We are now in a position to give a characterisation of the commutative von Neumann algebras we are interested in. For $J \in \mathcal{T}$ the algebras $\mathcal{A}_{P_{J}}$ are formally defined by

$$
\mathcal{A}_{P_{J}}=\left\{M\left(T_{J}\right): M \in L^{\infty}(\mathbb{R})\right\} \text {. }
$$

For general $P \in \mathcal{Q}$ take $\tau \in G$ which conjugates $P$ to one of the $P_{J}$ and put

$$
\mathcal{A}_{P}=\omega_{\tau} \mathcal{A}_{P_{J}} \omega_{\tau}^{*}
$$

ThEOREM 3.1. Let $P \in \mathcal{Q}$ and let $T$ be a bounded operator on $L^{2}(\mathbb{R})$. Assume that $T$ is $D$-class with respect to the decomposition of $L^{2}(\mathbb{R})$ induced by $P$ and let $\sigma \in \mathcal{S}^{\prime}\left(\mathbb{R}^{2}\right)$ be such that $T=\sigma^{w}$. The following conditions are equivalent:

(i) $T \in \mathcal{A}_{P}$,

(ii) $T$ commutes with $U_{t}^{P}$,

(iii) $\sigma$ is constant on the level lines of $P$.

Proof: The proof follows by reduction to the five basic cases. The cases $e, l, d$ are known. The proof of the cases $h, p$ follows from Propositions 3.5, 3.6 and the fact that operators with even kernels have even Weyl symbols.

Our next target is to give a description of the multiplier-symbol correspondence. We do it by means of the Wigner distribution. This correspondence is well-known in the cases $e, l, d$, as we now recall. 
The Wigner distribution $W_{e_{m}}$ is:

$$
W_{e_{m}}(x, \xi)=2(-1)^{m} L_{m}^{(0)}\left(4 \pi|z|^{2}\right) e^{-2 \pi|z|^{2}},
$$

where $z=(x, \xi)$ and $L_{m}^{(0)}=\sum_{k=0}^{m}\left((m !) /\left((m-k) !(k !)^{2}\right)\right)(-x)^{k}$ is the $j^{\text {th }}$ Laguerre polynomial. As for $\ell_{\lambda}, W_{l_{\lambda}} \in \mathcal{S}^{\prime}\left(\mathbb{R}^{2}\right)$ and for $F \in \mathcal{S}\left(\mathbb{R}^{2}\right)$

$$
W_{l_{\lambda}}(F)=\int_{-\infty}^{\infty} F(x, \lambda) d x
$$

For a bounded, measurable $M$ we denote by $\sigma_{J}(M)$ the tempered distribution corresponding to $M\left(T_{J}\right)$, that is, the distribution for which $\sigma_{J}^{w}(M)=M\left(T_{J}\right)$. The symbols which correspond to the multiplier $M$ have the form:

$$
\begin{aligned}
\sigma_{e}(M)(F) & =\sum_{m} M(m) W_{e_{m}}(F) \\
& =\sum_{m} M(m)(-1)^{m} 2 \pi \int_{0}^{\infty} F_{r}(s) L_{m}^{(0)}(4 \pi s) e^{-2 \pi s} d s
\end{aligned}
$$

where $F_{r}(s)=1 /(2 \pi) \int_{0}^{2 \pi} F\left(s e^{i t}\right) d t$

$$
\sigma_{l}(M)(F)=\int_{-\infty}^{\infty} M(\lambda) W_{l_{\lambda}}(F) d \lambda=\int_{-\infty}^{\infty} M(\lambda) \int_{-\infty}^{\infty} F(x, \lambda) d x d \lambda
$$

In the first cases the symbol is given by the expansion in terms of Laguerre functions. In the second and the third cases the symbols are constant on the horizontal lines $\xi=\lambda$ and take the values $M(\lambda), M\left(\lambda^{2}\right)$ on them.

Our final target is to complete the list of multiplier-symbol correspondences in the remaining cases. We start by computing Wigner distributions in the cases $h$ and $p$.

Proposition 3.7. Let $h_{\lambda}^{+}(x)=h_{\lambda}(x)$ for $x>0,0$ for $x<0$, and $h_{\lambda}^{-}(x)=$ 
$h_{\lambda}^{+}(-x)$. For $F \in \mathcal{S}\left(\mathbb{R}^{2}\right)$ we have

$$
\begin{aligned}
& W_{h_{\lambda}^{+}}(F)=\frac{1}{\lambda} \int_{-\infty}^{\infty} \int_{-\infty}^{\infty} e^{(x+y) / 2}\left(\mathcal{F}_{2} F\right) \\
& \quad \times\left(\frac{e^{x}+e^{y}}{2}, e^{x}-e^{y}\right) e^{-2 \pi i(\log \lambda)(y-x)} d x d y \\
& W_{h_{\lambda}^{-}}(F)=\frac{1}{\lambda} \int_{-\infty}^{\infty} \int_{-\infty}^{\infty} e^{(x+y) / 2}\left(\mathcal{F}_{2} F\right) \\
& \quad \times\left(-\frac{e^{x}+e^{y}}{2},-e^{x}+e^{y}\right) e^{-2 \pi i(\log \lambda)(y-x)} d x d y
\end{aligned}
$$

$$
\begin{aligned}
W_{p_{\lambda}}(F)=\int_{-\infty}^{\infty} \int_{-\infty}^{\infty} e^{2 \pi i\left(\left(x^{3} / 3\right)-\left(y^{3} / 3\right)\right)}\left(\mathcal{F}_{2} F\right) \\
\quad \times\left(\frac{x+y}{2}, x-y\right) e^{-2 \pi i \lambda(y-x)} d x d y,
\end{aligned}
$$

where $\mathcal{F}_{2}$ denotes the Fourier transform with respect to the second variable.

Proof: This follows easily from the definition of the Wigner distribution on $\mathcal{S}^{\prime}(\mathbb{R})$.

In the final formulation of our result we shall need the following map $H^{\prime}$.

Proposition 3.8. The map

$$
H F=\frac{1}{\cosh (\cdot / 2)} F\left(-2 \tanh \frac{\dot{2}}{2}\right)
$$

transforms $\mathcal{S}(\mathbb{R})$ into $\mathcal{S}(\mathbb{R})$. Its adjoint $H^{\prime}$, defined by the equation $H^{\prime} M(F)=$ $M(H F)$, where $M \in \mathcal{S}^{\prime}(\mathbb{R})$ and $F \in \mathcal{S}(\mathbb{R})$, maps $\mathcal{S}^{\prime}(\mathbb{R})$ into $\mathcal{S}^{\prime}(\mathbb{R})$. If $M$ is a polynomially bounded function then

$$
H^{\prime} M(F)=\int_{-2}^{2}\left(1-\left(\frac{x}{2}\right)^{2}\right)^{-1 / 2} M\left(\log \frac{1-(x / 2)}{1+(x / 2)}\right) F(x) d x .
$$

For $P \in \mathcal{Q}$ the symbol $T_{P}$ denotes the selfadjoint extension of $P^{w}$ defined in terms of the spectral measure corresponding to $P$.

THEOREM 3.2. Let $P \in \mathcal{Q}$ and let $\tau \in G$ be an affine transformation bringing $P$ to the form $P_{J}$, that is,

$$
P=\lambda P_{J} \circ \tau^{-1}+c, \lambda, c \in \mathbb{R}, J \in \mathcal{T} .
$$

Given a bounded function $M$, let $\sigma_{P}(M)$ be the tempered distribution for which $\sigma_{P}(M)^{w}=M\left(T_{P}\right)$. One may express $\sigma_{P}(M)$ in terms of $M$ as follows:

$$
\sigma_{P}(M)=\sigma_{J}\left(M_{\lambda, c}\right) \circ \tau^{-1}, \text { where } M_{\lambda, c}(x)=M(\lambda x+c) .
$$


For $F \in \mathcal{S}\left(\mathbb{R}^{2}\right)$ and $J \in\{e, l, d\}, \sigma_{J}(M)(F)$ is given by formulas (3.5), (3.6) and (3.7), respectively, while for $J \in\{h, p\}$ :

$$
\begin{aligned}
\sigma_{h}(M)(F) & =\int_{0}^{\infty} M(\log \lambda)\left(W_{h_{\lambda}^{+}}(F)+W_{h_{\lambda}^{-}}(F)\right) d \lambda \\
& =\lim _{n \rightarrow+\infty} \lim _{r \rightarrow 0} \mathcal{F} H^{\prime} \mathcal{F} M_{n}\left(\int_{0}^{\infty} F_{r}\left(t, \frac{\dot{t}}{t}\right) \frac{d t}{t}+\int_{0}^{\infty} F_{r}\left(-t,-\frac{\dot{t}}{t}\right) \frac{d t}{t}\right),
\end{aligned}
$$

where $F_{r}(x, \xi)=(1-h(x / r))(1-h(\xi / r)) F(x, \xi), h \in C_{c}^{\infty}(\mathbb{R}), 0 \leqslant h \leqslant 1, h \equiv 1$ on some neighbourhood of $0, M_{n}(x)=M(x) \chi_{(-n, n)}(x)$ and

$$
\begin{aligned}
\sigma_{p}(M)(F) & =\int_{-\infty}^{\infty} M(\lambda) W_{p_{\lambda}}(F) d \lambda \\
& =\mathcal{F}^{-1} e^{-2 \pi i \cdot 3 / 12} \mathcal{F} M\left(\int_{-\infty}^{\infty} F\left(u, \cdot+u^{2}\right) d u\right)
\end{aligned}
$$

Proof: Since $P=\lambda P_{J} \circ \tau^{-1}+c$, we get $T_{P}=\omega_{\tau}\left(\lambda T_{J}+c I\right) \omega_{\tau}^{-1}$. It follows that

$$
\begin{aligned}
\sigma_{P}(M)^{w} & =M\left(T_{P}\right)=\omega_{\tau} M\left(\lambda T_{J}+c I\right) \omega_{\tau}^{-1} \\
& =\omega_{\tau} M_{\lambda, c}\left(T_{J}\right) \omega_{\tau}^{-1}=\left(\sigma_{J}\left(M_{\lambda, c}\right) \circ \tau^{-1}\right)^{w}
\end{aligned}
$$

Clearly (3.14) proves (3.11).

Let $f, g \in \mathcal{S}(\mathbb{R})$. The orthogonality relations for the Wigner distribution and the spectral representation of $T_{h}$ imply

$$
\begin{aligned}
& \int_{0}^{\infty} M(\log \lambda)\left(W_{h_{\lambda}^{+}}\left(W_{f, g}\right)+W_{h_{\lambda}^{-}}\left(W_{f, g}\right)\right) d \lambda \\
& \quad=\int_{0}^{\infty} M(\log \lambda)\left(\left\langle f, h_{\lambda}^{+}\right\rangle\left\langle h_{\lambda}^{+}, g\right\rangle+\left\langle f, h_{\lambda}^{-}\right\rangle\left\langle h_{\lambda}^{-}, g\right\rangle\right) d \lambda \\
&=\left\langle M\left(T_{h}\right) f, g\right\rangle .
\end{aligned}
$$

Formula (3.15) proves the first equality in (3.12). In order to prove the second equality in (3.12), put

$$
L_{r}(x, y)=e^{(x+y) / 2}\left(\mathcal{F}_{2} F_{r}\right)\left(\frac{e^{x}+e^{y}}{2}, e^{x}-e^{y}\right)
$$

and for any positive integer $n$ let $M_{n}(t)=M(t) \chi_{(-n, n)}(t)$. Since $M_{n}(t) \rightarrow M(t)$ pointwise and $M_{n} \in L^{1}(\mathbf{R})$, by applying (3.8) and dominated convergence twice we 
obtain the following equalities

$$
\begin{aligned}
\int_{0}^{\infty} M(\log \lambda) W_{h_{\lambda}^{+}}(F) d \lambda= & \lim _{n \rightarrow+\infty} \int_{-\infty}^{\infty} M_{n}(t) \int_{-\infty}^{\infty} \int_{-\infty}^{\infty} e^{(x+y) / 2} \\
& \times\left(\mathcal{F}_{2} F\right)\left(\frac{e^{x}+e^{y}}{2}, e^{x}-e^{y}\right) e^{-2 \pi i t(y-x)} d x d y d t \\
= & \lim _{n \rightarrow+\infty} \lim _{r \rightarrow 0} \int_{-\infty}^{\infty} M_{n}(t) \int_{-\infty}^{\infty} \int_{-\infty}^{\infty} L_{r}(x, y) \\
& \times e^{-2 \pi i t(y-x)} d x d y d t .
\end{aligned}
$$

Observe that for any $L \in \mathcal{S}\left(\mathbb{R}^{2}\right)$

$$
\begin{aligned}
\int_{-\infty}^{\infty} \int_{-\infty}^{\infty} \int_{-\infty}^{\infty} L(x, y) e^{-2 \pi i t(y-x)} d x d y e^{2 \pi i t \xi} d t & \\
& =\int_{-\infty}^{\infty} L\left(u-\frac{\xi}{2}, u+\frac{\xi}{2}\right) d u:=\psi_{L}(\xi)
\end{aligned}
$$

so that, on applying the Fourier transform to both sides of (3.17), the expression inside the double limit in (3.16) becomes

$$
\begin{aligned}
& \int_{-\infty}^{\infty} M_{n}(t)\left(\mathcal{F} \psi_{L_{r}}\right)(t) d t=\int_{-\infty}^{\infty}\left(\mathcal{F} M_{n}\right)(t) \psi_{L_{r}}(t) d t \\
& =\mathcal{F} M_{n}\left(\int_{0}^{+\infty} \mathcal{F}_{2} F_{r}\left(t \cosh \frac{\dot{\tau}}{2},-2 t \sinh \frac{\dot{j}}{2}\right) d t\right) \\
& =\mathcal{F} M_{n}\left(\int_{0}^{\infty} \mathcal{F}_{2} F_{r}\left(t,-2 t \tanh \frac{\dot{\gamma}}{2}\right) \frac{d t}{\cosh (\cdot / 2)}\right) \text {. }
\end{aligned}
$$

Now, since $x \mapsto \int_{0}^{+\infty} \mathcal{F}_{2} F_{r}(t, t x) d t$ is a Schwartz function, by applying Proposition (3.8) to (3.18) we obtain

$$
\begin{aligned}
\int_{-\infty}^{\infty} M_{n}(t)\left(\mathcal{F} \psi_{L_{r}}\right)(t) d t & =H^{\prime} \mathcal{F} M_{n}\left(\int_{0}^{\infty} \mathcal{F}_{2} F_{r}(t, t \cdot) d t\right) \\
& =\mathcal{F} H^{\prime} \mathcal{F} M_{n}\left(\int_{0}^{\infty} F_{r}\left(t, \frac{\dot{t}}{t}\right) \frac{d t}{t}\right)
\end{aligned}
$$

Clearly, combining this with (3.15) and (3.16) the second equality in (3.12) follows, whereby one uses (3.9) in place of (3.8) to treat $W_{h_{\lambda}^{-}}(F)$.

The proof of the first equality in (3.13) goes similarly as in the case of (3.12). As for the proof of the second equality in (3.13), an application of (3.10) and (3.17) gives 
the following expression for the integral $\int_{-\infty}^{\infty} M(\lambda) W_{p_{\lambda}}(F) d \lambda$ :

$$
\begin{aligned}
\int_{-\infty}^{\infty} M(\lambda) \int_{-\infty}^{\infty} \int_{-\infty}^{\infty} e^{2 \pi i\left(\left(x^{3} / 3\right)-\left(y^{3} / 3\right)\right)} \mathcal{F}_{2} F\left(\frac{x+y}{2}, x-y\right) e^{-2 \pi i \lambda(y-x)} d x d y d \lambda \\
=\mathcal{F} M\left(\int_{-\infty}^{\infty} e^{2 \pi i\left((u-(\cdot / 2))^{3} / 3\right)-\left((u+(\cdot / 2))^{3} / 3\right)} \mathcal{F}_{2} F(u,-\cdot) d u\right) \\
=e^{-2 \pi i\left(\cdot^{3} / 12\right)} \mathcal{F} M\left(\int_{-\infty}^{\infty} e^{-2 \pi i u^{2} \cdot} \mathcal{F}_{2} F(u,-\cdot) d u\right) \\
=\mathcal{F}^{-1} e^{-2 \pi i(\cdot 3 / 12)} \mathcal{F} M\left(\int_{-\infty}^{\infty} F\left(u, \cdot+u^{2}\right) d u\right) .
\end{aligned}
$$

Comments. (i) The letters $e, h, d, p, l$ stand for elliptic, hyperbolic, degenerate, parabolic, linear and refer to the geometric loci associated to the corresponding polynomials. The orbits of the one-parameter subgroups $g_{t}^{J}$ and the level lines of $P_{J}$ are, respectively: circles centred at the origin, hyperbolas, horizontal lines, parabolas and again horizontal lines.

(ii) The derivatives of phase functions are called istantaneous frequencies. They indicate what the frequency is at a given time. The graphs of the istantaneous frequencies of the systems $h_{\lambda}, e_{\lambda}, p_{\lambda}$ are hyperbolas, horizontal lines and parabolas.

(iii) In the hyperbolic case the graphs of the istantaneous frequencies corresponding to $\mathcal{H}_{K}$ fill out the whole quadrant $K$. Thus the quadrant $K$ corresponds to $\mathcal{H}_{K}$. In the degenerate case the domains corresponding to $\mathcal{D}_{I}, \mathcal{D}_{I I}$ are the upper and lower half planes.

(iv) All of the distributions $\sigma_{P}(M)$ involve geometric ingredients, that is integrals over the level lines of $P$.

(v) Let $f \in C_{c}(\mathbb{R})$ be fixed. For $P \in \mathcal{Q}, \phi \in L^{2}(\mathbb{R})$ define the operator

$$
T_{P, \phi} h(x)=f(x)\left\langle h, U_{x}^{P} \phi\right\rangle .
$$

The operator $T_{P, \phi}^{*} T_{P, \phi}$ is simply an average of one dimensional projections on the vector $\omega_{g_{t}^{p}} \phi$. One can show (see [11]), that for all $0<p \leqslant \infty$

$$
\left\|T_{P, \phi}\right\|_{S^{p}}^{p} \cong \sum_{n}\left\|\chi_{[n, n+1]}\left(T_{P}\right) \phi\right\|_{L^{2}}^{p}
$$

$S^{p}$ denotes the $p$-Schatten class. The operator $\chi_{[n, n+1]}\left(T_{P}\right)$ is interpreted as a restriction to the region $n \leqslant P(x, \xi) \leqslant n+1$ of the time-frequency plane.

\section{REFERENCES}

[1] R. Baraniuk, Shear madness: signal-dependent and metaplectic time-frequency representations, (Ph.D. Thesis) (University of Illinois, Urbana-Champaign, 1992). 
[2] I. Daubechies, Ten lectures on wavelets (CBMS-NSF Regional Conference Series, no. 6, SIAM, Philadelphia, 1992).

[3] F. De Mari and K. Nowak, 'Canonical subgroups of $\mathbb{H}_{1} \rtimes S L(2, \mathbb{R})$ ', Boll. Un. Mat. Ital. (to appear).

[4] H.G. Feichtinger and K. Gröchenig, 'Gabor wavelets and the Heisenberg group; Gabor expansions and short time Fourier transform from the group theoretical point of view', in Wavelets - A Tutorial in Theory and Applications, (C.K. Chui, Editor) (Academic Press, Boston, 1992), pp. 359-397.

[5] H.G. Feichtinger and T. Strohmer, Eds., Gabor analysis and algorithms - Theory and applications, Applied and Numerical Harmonic Analysis (Birkhauser Boston, Boston, MA, 1997).

[6] G.B. Folland, Harmonic analysis in phase space, Annals of Mathematics Studies (Princeton University Press, Princeton, N.J., 1989).

[7] G.B. Folland and A. Sitaram, 'The uncertainty principle: A mathematical survey', $J$. Fourier Anal. Appl. 3 (1997), 207-237.

[8] M. Frazier, B. Jawerth and G. Weiss, Littlewood-Paley theory and the study of function spaces, CBMS Regional Conference Series in Mathematics 79 (American Mathematical Society, Providence, R.I., 1991).

[9] L. Hörmander, The analysis of linear partial differential operators III (Springer-Verlag, Berlin, Heidelberg, New York, Tokyo, 1985).

[10] Y. Meyer, Wavelets, algorithms and applications (SIAM, Philadelphia, PA, 1993).

[11] K. Nowak, 'Certain function spaces related to the metaplectic representation', Collog. Math. 76 (1998), 143-152.

\section{DIMET}

Piazzale J. F. Kennedy, Pad. D

16129 Genova

Italy

e-mail: demari@dima.unige.it
Purchase College

735 Anderson Hill Rd.

Purchase, NY 10577-1400

United States of America

e-mail: knowak@gmx.de 